\title{
Organizational model for the management of patients with chronic allergic conditions
}

\author{
Edyta Krzych-Fałta ${ }^{1}$, Mira Lisiecka-Biełanowicz ${ }^{1}$, Konrad Furmańczyk ${ }^{1,2}$, Barbara Piekarska ${ }^{1}$, Oksana Wojas ${ }^{1}$, \\ Bolesław Samoliński ${ }^{1}$
}

${ }^{1}$ Department of the Environmental Hazards Prevention and Allergology, Faculty of Health Sciences, Medical University of Warsaw, Warsaw, Poland

2Faculty of Applied Informatics and Mathematics, Warsaw University of Life Sciences, Warsaw, Poland

Adv Dermatol Allergol 2019; XXXVI (3): 339-345 DOI: https://doi.org/10.5114/ada.2019.84288

\begin{abstract}
Introduction: Allergic conditions present a serious problem for the Polish population. The most common form of allergy is allergic rhinitis. Due to its chronic nature and a complex pathophysiological mechanism the allergic rhinitis could be associated with others comorbidities. That is why it is a poses a considerable bio-psychosocial problem both for the affected individual patient and for the healthcare system.

Aim: To identify organizational factors and analyse their effect on patients with chronic allergic conditions.

Material and methods: The study group comprised 9,370 respondents (including 1,967 patients with allergic rhinitis (AR), 372 patients with self-reported bronchial asthma (BA1), and 351 patients with physician-diagnosed bronchial asthma (BA2)) aged from 20 to 44 years, some of whom were additionally examined (skin pick tests) in an outpatient setting. The findings obtained in the outpatient subgroup $(n=4,783)$ were analysed in more detail. The study was based on translated and validated ECRHS and ISAAC questionnaires.

Results: Over a half of the evaluated patients with AR visited a specialist due to their symptoms. Some respondents neither visited their doctor regularly nor had regular check-ups, which undoubtedly affected their quality of life. Conclusions: The authors propose an organizational model incorporating the position of a healthcare educator (HE) as a coordinator in the process of managing chronic allergic conditions in adult patients within the Polish healthcare system. Adult patient education by the HE would include introducing the principles of secondary and tertiary prevention in order to improve the quality of life in patients with chronic allergic conditions.
\end{abstract}

Key words: allergies, organizational model, healthcare educator.

\section{Introduction}

Allergic conditions present a serious problem for social medicine and public health. An estimated near 40\% of the general Polish population have been diagnosed with an allergic condition [1]. The most common form of allergy is allergic rhinitis (AR). This inflammatory condition has a complex pathophysiological mechanism; this is why it is associated with such comorbidities as allergic conjunctivitis, sinusitis, nasal polyps, adenoid hypertrophy, exudative otitis media, gastrointestinal reflux, and bronchial asthma (BA). Due to its chronic nature and exhausting symptoms, BA poses a considerable bio-psychosocial problem both for the affected individuals and for the healthcare system. In addition, $66.9 \%$ of all respondents demonstrated a lack of awareness that they suf- fered from BA (undiagnosed BA) and 39\% of those who believed to be affected turned out to have been overdiagnosed with BA [2].

In light of the above, early diagnosis, a relatively promptly introduced treatment, and (depending on the society's needs) concurrent secondary or tertiary prevention, with the key role of a healthcare educator (HE), seem crucial.

The authors propose an organizational model for patient care involving an $\mathrm{HE}$ in the management of adult patients with chronic allergic conditions. In this model, the HE acts a coordinator whose healthcare tasks required in managing patients with chronic allergic conditions include [3]:

- helping patients get accustomed to the new life situation associated with a chronic allergic condition;

Address for correspondence: Edyta Krzych-Fałta PhD, Department of the Environmental Hazards Prevention and Allergology, Faculty of Health Sciences, Medical University of Warsaw, 1 a Banach St, 02-097 Warsaw, Poland, phone: +48 22599 10 40, e-mail: e.krzych@gmail.com Received: 15.03.2018, accepted: 9.04.2018. 
- understanding and developing a novel, constructive approach to patients' identified needs;

- educating patients about their condition and the associated limitations.

Implementing their knowledge on the use of the available devices (e.g. by acquiring practical skills in monitoring and measuring the parameters of their own chronic allergic condition) may help chronic-allergy patients achieve a satisfactory quality of life.

\section{Aim}

The purpose of this study was to identify organizational factors and analyse their effect on patients with chronic allergic conditions (more specifically on adult AR and BA patients exposed to mould allergens at home), in terms of how often they seek specialist consultation.

\section{Material and methods}

This study was conducted in a Polish population randomly selected by the Ministry of Interior and Administration from the national PESEL (personal identification number) database. The tools used in the study were the European Community Respiratory Health Survey (ECRHS) [4] and International Study of Asthma and Allergies in Childhood (ISAAC) [5] questionnaires, adapted to European conditions (Middle and Eastern Europe) and used as part of the Implementation of a System for the Prevention and Early Detection of Allergic Diseases in Poland (ECAP) [1] study. The ECAP study was conducted in eight metropolitan areas (Gdansk, Wroclaw, Poznan, Katowice, Krakow, Lublin, Bialystok, Warszawa) and in rural areas (Krasnostawski county) and comprised two stages. In the first stage, all respondents $(n=18,617)$ were screened based on a survey, with the help of the Personal Data Assistant (PDA) tool. The second stage, which was conducted in an outpatient setting, included 4,783 eligible respondents, who underwent complementary assessments: skin prick tests (birch, grasses/cereals, Dermatophagoides pteronyssinus and Dermatophagoides farinae, moulds (set 1 comprising: Botrytis cinerea, Cladosporium herbarum, Alternaria tenuis, Curvularia lunata, Fusarium moniliforme, Helminthosporium; and set 2 comprising: Aspergillus fumigatus, Mucor mucedo, Penicillium notatum, Pullularia pullulans, Rhizopus nigricans, Serpula lacrymans), cat, dog, control solution, histamine), lung function tests, and peak nasal inspiratory flow (PNIF). The diagnoses of self-reported AR and BA were verified based on the criteria presented by the Allergic Rhinitis and its Impact on Asthma (ARIA) [6] initiative and Global Initiative for Asthma (GINA) program [7].

In line with the purpose of the study, 9,370 patients were included in analysis. This group included patients with self-reported $\operatorname{AR}(n=1,967 ; 21 \%)$, patients with self-reported bronchial asthma (BA1) $(n=372 ; 4 \%)$, and patients with physician-diagnosed bronchial asthma (BA2) ( $n=531 ; 4 \%)$; subgroups AR and BA1/BA2 were not mutually exclusive (Table 1). Some respondents were additionally examined (skin pick tests) in an outpatient setting. The findings obtained in the outpatient subgroup $(n=4,783)$ were analysed in more detail.

Both sex subgroups were similar in terms of age, with the mean age of 31.9 years in the female group and 31.3 years in the male group. In total, females constituted $59 \%(n=5,518)$ and males $41 \%(n=3,868)$ of the analysed study population. Metropolitan-area inhabitants constituted $89 \%$ of the analysed population $(n=8,337)$, while rural-area inhabitants were a much smaller group ( $n=1,049 ; 11 \%)$.

The study was approved by the Medical University of Warsaw Institutional Review Board (KB/206/2005) and Personal Data Protection Office.

Table 1. Analysed parameters (questions) and the abbreviations used

\begin{tabular}{ll}
\hline Q1 & Have you ever spent a night in a hospital because of breathing problems? \\
\hline Q2 & Did you spend a night in a hospital because of breathing problems in the last 12 months? \\
\hline Q3 & Have you ever been seen by a doctor because of breathing problems or because of shortness of breath? \\
\hline Q4 & Were you seen by a general practitioner because of breathing problems or shortness of breath in the last 12 months? \\
\hline Q5 & $\begin{array}{l}\text { Did you see a specialist (chest physician, allergy specialist, internal medicine specialist, ENT doctor) because of your breathing } \\
\text { problems or shortness of breath in the last } 12 \text { months? }\end{array}$ \\
\hline Q6 & Are you given regular appointments to be seen by a doctor (or nurse) for your asthma, wheezing or shortness of breath? \\
\hline QA & $\begin{array}{l}\text { Within the last } 12 \text { months did you have wet or damp spots on surfaces inside your home other than in the basement } \\
\text { (for example on walls, wall paper, ceilings or carpets)? }\end{array}$ \\
\hline QB & Has there ever been any mould or mildew on any surface, other than food, inside the home? \\
\hline QC & Was there mould or mildew on any surfaces inside the home in the last 12 months? \\
\hline AR & Allergic rhinitis subgroup \\
\hline BA1 & Self-reported bronchial asthma subgroup \\
\hline BA2 & Physician-diagnosed bronchial asthma subgroup \\
\hline
\end{tabular}




\section{Statistical analysis}

Statistical analysis involved rate calculation and the use of R's prop.test for testing if the proportions in several groups were the same. The $p$-value of 0.05 was considered statistically significant.

\section{Results}

Due to the need for implementing measures for improving the health of the Polish population, we analysed selected indoor factors (including the presence of mould inside the house) on experiencing allergy symptoms, with the latter evidenced by the rates of seeking specialist medical help.

\section{Respondents with allergic rhinitis}

Our analysis showed that patients with AR who were allergic to moulds reported observing the following phenomena on the walls and/or ceilings of their households: nearly $21 \%$ of them $(n=418)$ reported damp spots and $27 \%(n=522)$ reported mould, with $61 \%$ of the latter subgroup $(n=321)$ reporting the presence of mould within the previous 12 months. Here are our findings stratified by allergen types: the proportions of AR patients allergic to Cladosporium herbarum ( $n=78,13 \%$ of the subpopulation examined in an outpatient setting) who reported observing damp spots and mould in their households were $21 \%(n=16)$ and $28 \%(n=22)$, respectively, with $59 \%$ of the latter subgroup $(n=13)$ reporting mould within the last 12 months; the proportions of AR patients allergic to Alternaria tenuis ( $n=78 ; 13 \%$ of the AR patients examined in an outpatient setting) who reported damp spots and mould in their households were $17 \%(n=13)$ and $21 \%(n=16)$, respectively, with $50 \%$ of

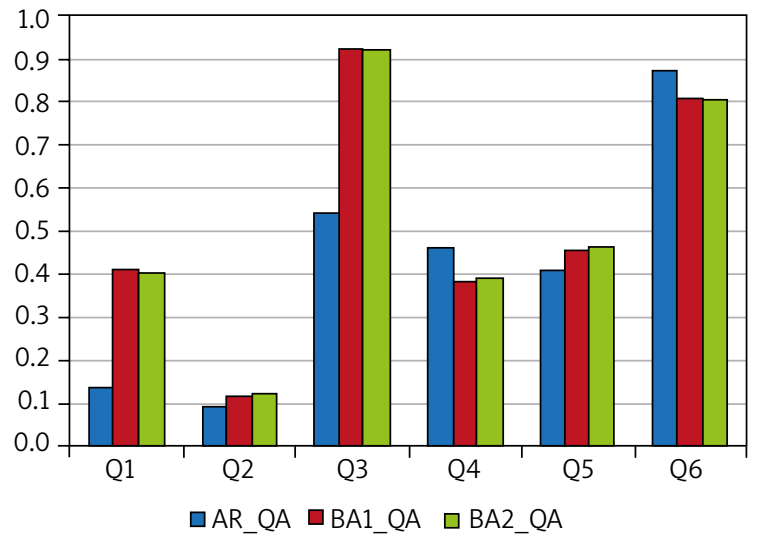

Figure 1. The proportions of those who answered "Yes" to individual questions Q1, Q2, Q3, Q4, Q5, and Q6 out of the respondents who answered "Yes" to question QA: Within the last 12 months did you have wet or damp spots on surfaces inside your home other than in the basement (for example on walls, wall paper, ceilings or carpets)? the latter subgroup $(n=8)$ reporting mould within the last 12 months. The proportions of AR patients who reported observing damp spots/mould in their households were similar to those recorded in both the self-reported BA and physician-diagnosed BA subgroups (Figure 1).

\section{Respondents with self-reported bronchial asthma} (BA1)

Damp spots and mould in households were reported by $20 \%(n=74)$ and $31 \%(n=133)$ of these respondents, respectively, with the presence of mould within the previous 12 months observed by $57 \%$ of the latter subgroup $(n=64)$. Moreover, a total of $13 \%$ of those BA1 patients who were additionally examined in an outpatient setting $(n=10)$ had a diagnostically confirmed allergy to Cladosporium herbarum, while $14 \%(n=15)$ were allergic to Alternaria tenuis. Out of the patients allergic to Cladosporium herbarum, $30 \%$ and $60 \%$ reported damp spots and mould, respectively, with $25 \%$ of the latter reporting mould within the last 12 months. The proportions of patients allergic to Alternaria tenuis who reported damp spots and mould in their households were $7 \%(n=1)$ and $27 \%(n=4)$, respectively, with nearly $25 \%(n=1)$ of the latter subgroup reporting mould within the last 12 months (Figure 2).

\section{Respondents with physician-diagnosed bronchial asthma (BA2)}

Damp spots and mould in households were reported by $73(21 \%)$ and 109 (31\%) respondents from this group, respectively, with $57 \%$ of the latter subgroup $(n=62)$ reporting mould within the previous 12 months. Moreover, out of those BA2 patients who were examined in an outpatient setting 9 (9\%) were allergic to Cladosporium herbarum. Out of those, 3 (33\%) and 4 (56\%) pa-

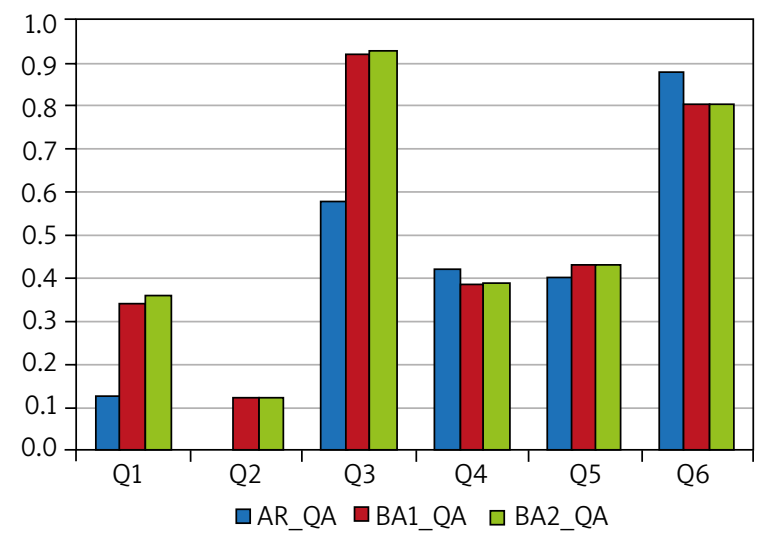

Figure 2. The proportions of those who answered "Yes" to individual questions Q1, Q2, Q3, Q4, Q5, and Q6 out of those respondents who answered "Yes" to question QB: Has there ever been any mould or mildew on any surface, other than food, inside the home? 
tients reported damp spots and mould, respectively, with $25 \%(n=1)$ of the latter reporting mould within the last 12 months. Out of those BA2 patients who were examined in an outpatient setting $14(13 \%)$ proved allergic to Alternaria tenuis. Out of those, 1 (7\%) and 4 (29\%) patients reported damp spots and mould, respectively, with $25 \%$ of the latter $(n=1)$ reporting mould within the last 12 months. There were significant differences in proportions of respondents with $A R, B A 1$, and $B A 2$ reporting damp spots in their households during the last 12 months who answered "Yes" to individual questions Q1-Q6 ( $p<0.001)$. The subgroups of patients with AR, $B A 1$, and BA2 who reported mould in their households differed significantly in terms of the proportions of respondents who answered "Yes" to individual questions Q1-Q6 ( $p<0.001)$. There were significant differences in the rates of responses "Yes" to questions Q1-Q6 among those from AR, BA1, and BA2 subgroups who reported mould in their households within the last 12 months ( $p<0.001)$ (Figure 3).

Over a half of respondents with AR visited a medical specialist due to shortness of breath. Respondents with self-reported BA as well as those with physician-diagnosed BA reported seeing a doctor. Interestingly, patients from none of the evaluated subgroups (AR, self-reported $B A$, and physician-diagnosed BA) had regular physical exams or doctor appointments. We would like to emphasize that over $80 \%$ of patients with AR or BA1/BA2 were not given regular appointments to see a doctor (question Q6). Moreover, there was a considerable variation in the rates of "Yes" responses to questions Q1 (on spending a night in a hospital because of breathing problems) and Q3 (on being seen by a doctor because of breathing problems or shortness of breath) between subgroups classified according to the type of condition (AR vs. BA1/

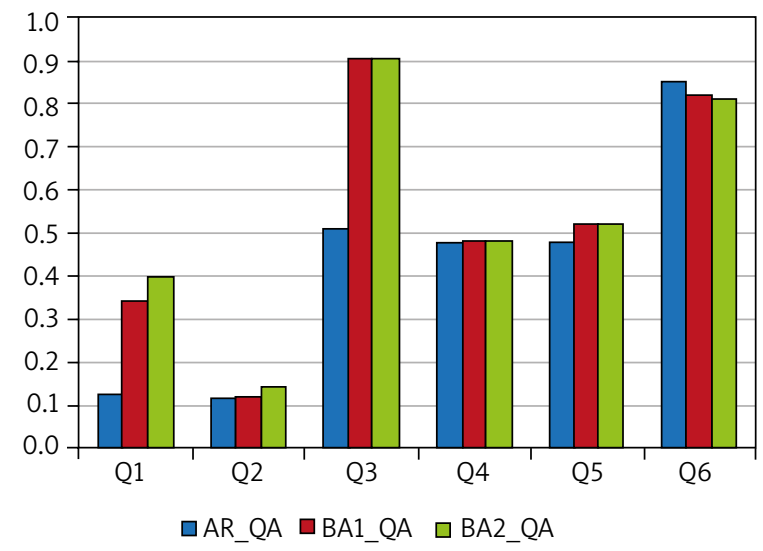

Figure 3. The proportions of those who answered "Yes" to individual questions Q1, Q2, Q3, Q4, Q5, and Q6 out of those respondents who answered "Yes" to question QC: Was there mould or mildew on any surfaces inside the home in the last 12 months?
BA2) $(p<0.001)$ among patients who reported mould in their households (QA, QB, and QC). Question Q1 was answered "Yes" by 10-15\% of those with AR and by $30-40 \%$ of those with BA1/BA2. Question Q3 was answered "Yes" by $50-55 \%$ of those with AR and by approximately $90 \%$ of those with BA1/BA2.

\section{Discussion}

Due to the nature of chronic allergic conditions (including the cyclic/seasonal occurrence and exacerbation of RA and BA symptoms), the management of patients suffering from such conditions requires indicated prevention efforts (when the risk of developing symptoms is already high). The recommended prevention efforts at this stage include specific measures that could avert symptoms by eliminating risk factors; this would eliminate or minimize the effects of the condition on the patients' lives or delay the onset of symptoms (at the same time delaying disease progression). The results of our study showed that over a half of patients with BA saw a medical specialist due to their symptoms (shortness of breath). Patients with both self-reported and physiciandiagnosed BA sought medical help. Interestingly, patients with BA (self-reported BA and physician-diagnosed BA) neither underwent regular (periodic) examinations nor had regular appointments to evaluate the symptoms of their allergic conditions. Is this situation due to the organization of care for adult patients with chronic allergic conditions? What is it that hinders the patients' access to a physician (or a medical specialist)?

The proposed new organizational model for managing patients with chronic allergic conditions aims to increase the involvement of the patients themselves and their families in the process of disease management, as well as to engage new entities (such as an HE). The process of managing patients with chronic allergic conditions is supplemented by information processing, including the processing of new data obtained from monitoring the health of patients with allergic conditions. A database-based system that contains the data from patient monitoring would provide information that could be used by a treatment expert, i.e. physician. Expert-based systems used in managing allergic conditions by means of repeated assessments of the chronic disease may offer an internet-based, interactive form of support (e.g. via telediagnostics) for the HE, as well as for the entire healthcare system. This additional, systemic control over the process of patient-executed health-centered actions would improve therapeutic effectiveness by ensuring that regular, scheduled doctor appointments are conducted or by increasing the patients' awareness of the options offered by the Polish healthcare organizations. The patients would be also equipped with various tools for the exchange of information, via educational and information websites, created specifically for patients and 
accessible with mobile devices (mobile Health; mHealth), as well as be able to exchange information and experiences through social media. It is important to consider the effect of organizational factors, including the patient's environment, chronic nature of the disease, and the structure of healthcare of the patient with a chronic allergic condition. Our study indicated that the lack of regular assessments for symptoms associated with allergic conditions was common both in those patients who reported noticing wet spots or mould on the walls of their homes in general and those who noticed these phenomena in the previous 12 months. We believe it is important to consider the components of the system of care provided to patients (including the support by the patient's family), patient's environment, and the chronic nature of the disease as these components/factors determine the course of chronic allergic conditions. These factors are listed in Figure 4. It seems important to introduce to the organization of the Polish healthcare system the coordination of various components of care for adult patients with chronic allergic conditions. The resulting model of care for adult patients with chronic allergic conditions would include:

The user (or patient); in contrast to the current, system-oriented solutions, the proposed model of care is based on knowledge management and collecting new data generated during patient monitoring, by combining the information obtained from various sources. Access to this knowledge and to other, complementary sources of psychological knowledge and the data on the patient's environment would help make diagnostic decisions in order to more effectively coordinate patient-oriented measures of secondary and tertiary prevention.

Organizational and technical solutions that involve combining the existing components of the Polish healthcare system and are oriented towards patients with chronic allergic conditions. This concept is based on the premise of providing the patient with support within the ecosystem, ensuring patient-activated use of information and communication technology (ICT) system resources, and offering support to individual patients by the HE via mobile technology tools, such as telediagnostics, mHealth, and social media. The ICT system helps monitor the course of chronic allergic conditions via various gauges, parameters, and factors (depending on the nature of the allergic condition, its course, and the patient's lifestyle). An example tool is an electronic Allergy Diary by MACVIA-ARIA; this application allows daily entries on the symptoms of AR ("hay fever") or asthma as well as medications.

A healthcare educator ( $\mathrm{HE})$, who - as a coordinator - has been positioned above the chronic allergy patient. The main goal of the HE would be to ensure the patients assume a more active role in the process of managing their disease and to support the patients in controlling their symptoms. HE's role would be contingent mostly

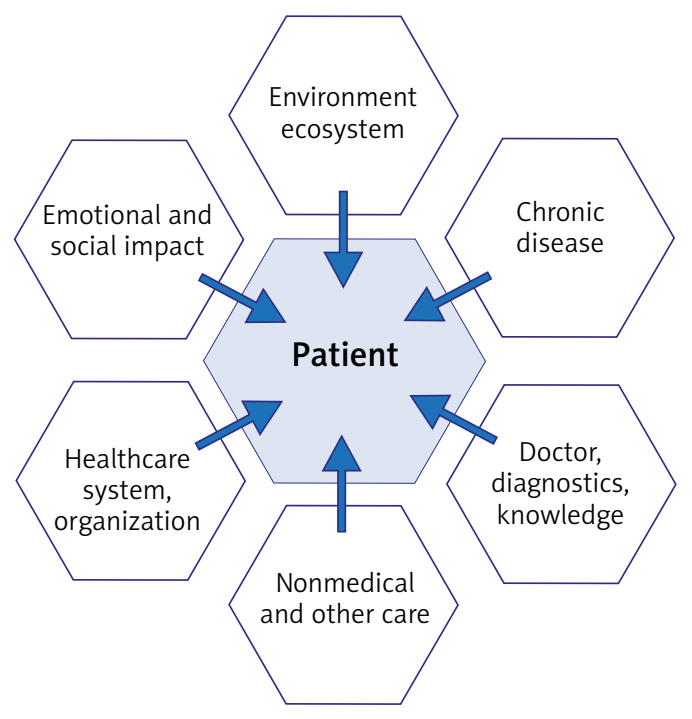

Figure 4. The key factors that affect patients with chronic disease with the current way the Polish healthcare system is organized [3]

on finding the right form of contact with the patient and ensuring adequate feedback. How patients manage their allergic conditions depends on the environmental factors present in their surroundings and patients' interactions with others (including the HE). Improving the quality of life of patients with chronic allergic conditions via the proposed model would require monitoring patients' health parameters and the environmental factors affecting the patients' surroundings.

\section{Conclusions}

Based on our assessments of the effect of selected mould allergens in the homes of adults with chronic allergic conditions (and the associated rates of seeking medical specialist advice) it is imperative to implement measures to prevent the associated health consequences in these patients. Improving the health of the Polish society requires that systemic changes be introduced to the care for adult patients with chronic allergic conditions [3]. Healthcare facilities included in the Polish hospital network [8] should organize integrated care for their patients [9]. The protocol for managing chronic conditions should ensure that high quality healthcare services be available at the optimal time over the course of treatment. Hence, we propose an organizational model that incorporates the position of the HE as a coordinator in the process of managing chronic allergic conditions in adult patients. This is illustrated in Figure 5. Patients with chronic allergic conditions exist in their own ecosystem, which is determined by the specific nature of the disease and specific treatment requirements as well as specific social aspects associated with the disease. Organizational and technical support by 


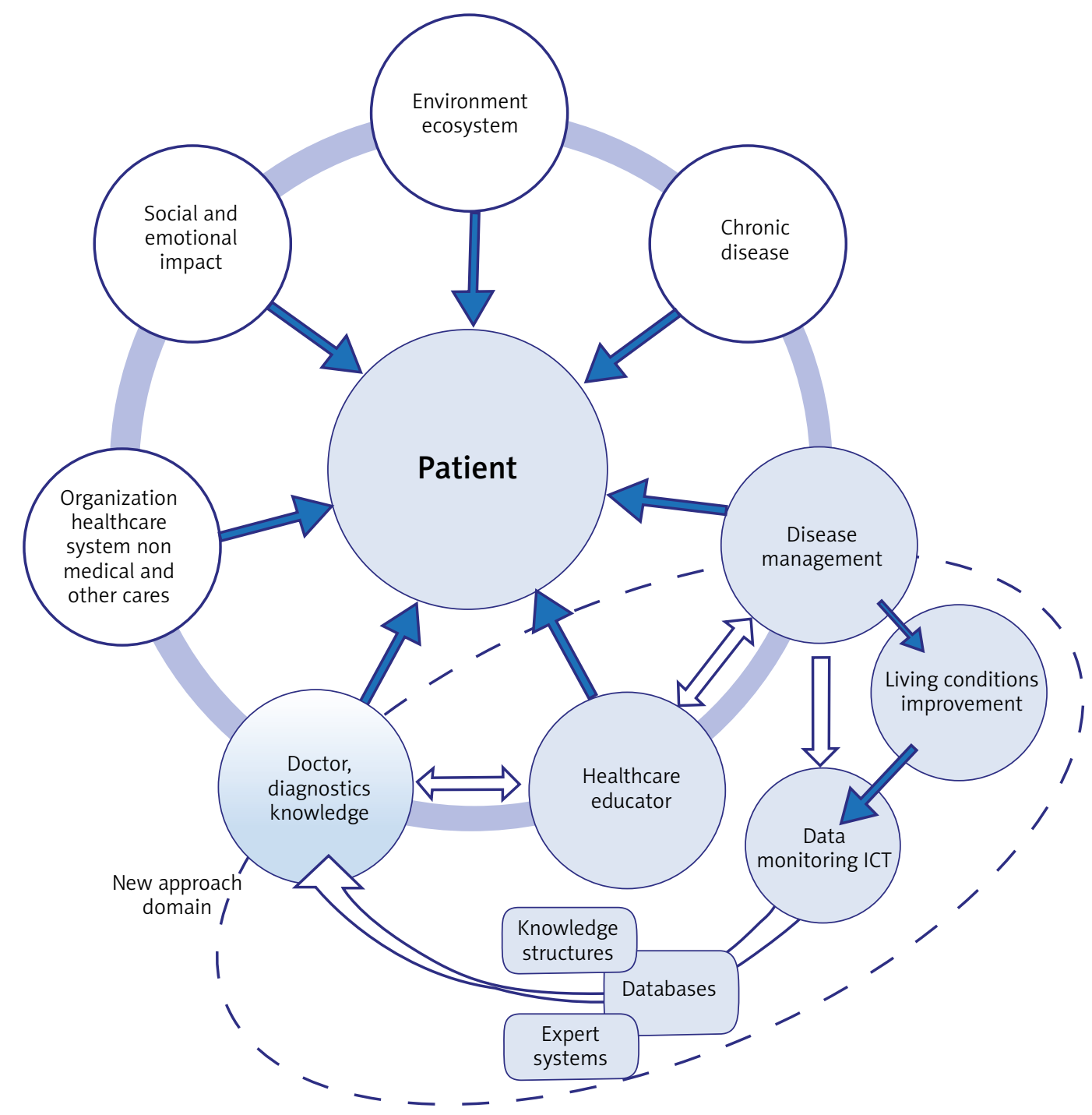

Figure 5. A healthcare model, which has been improved from the technical and organizational point of view, and is part of a patient-oriented healthcare ecosystem catering to patients with chronic conditions, in order to improve their quality of life [3]

the healthcare system and social networks plays an important role in the care for individual patients, as do the knowledge on the patient's chronic condition and having information on its course. Bases of such knowledge are going to be compiled with the use of data on chronic diseases, which may be used to continually improve the part of the Polish healthcare system dealing with individual care for patients with chronic diseases, including allergic conditions. The databases created this way may rely on various sources of information: both direct and indirect (such as demographic data, health data, and specialized databases for specific chronic conditions, including databases of monitored parameters).

The unique character of each chronic disease, including allergic conditions, requires engaging appropriate entities in the process of disease management. These entities include:

1. The HE, whose main role is to provide the patient with technical and emotional support as well as social stimulation. The health educator collaborates with the physician to help patients fully understand disease pathogenesis and to know both the possible complications and the causative or exacerbating factors (such as those in the patient's immediate environment).

2. The patient, who is co-responsible for the diagnostic and therapeutic process and for communicating with organizations within the healthcare system during the course of treatment and long-term care.

3. The healthcare organization which provides care for the patient and has the technical and organizational means for patient monitoring and measuring specific 
health-related parameters (in other words, the technical support via ICT tools and an information-exchange platform) as well as suitable logistics and various forms of organizational support, including maintaining constant communication with the patient.

Patients affected by allergic conditions and exposed to external factors would manage their own chronic disease as part of the healthcare system, with the active support from the HE, in order to improve their quality of life. One of the HE's roles is to make the patient aware of the need for continuously implementing preventive measures against chronic allergic conditions. Patient education measures used by the HE in dealing with adult patients would include introducing the principles of secondary and tertiary prevention of chronic allergic conditions.

\section{Conflict of interest}

The authors declare no conflict of interest.

\section{References}

1. Samoliński B, Raciborski F, Lipiec A, et al. Epidemiologia chorób w Polsce (ECAP). Pol J Allergol 2014; 1: 10-8.

2. Komorowski J. Epidemiologia astmy w Polsce w oparciu o wyniki badania ECAP. Thesis. Warsaw 2012.

3. Lisiecka-Biełanowicz M., Wawrzyniak Z. Healthcare model with use of information and communication technology for patients with chronic disease. Ann Agric Environ Med 2016; 23: 462-7.

4. European Community Respiratory Health Survey: variations in the prevalence of respiratory symptoms, self-reported asthma, and use of asthma madication in the European Community Respiratory Health Survey. Eur Respir J 1996; 9: 687-95.

5. The International Study of Asthma and Allergies in Childhood (ISAAC) Steering Committee: worldwide variation in prevalence of symptoms of asthma, allergic rhinoconjunctivitis, and atopic eczema. Lancet 1998; 351: 1225-32.

6. Brożek JL, Bousquet J, Agache I, et al. Allergic Rhinitis and its Impact on Asthma (ARIA) guidelines-2016 revision. J Allergy Clin Immunol 2017; 140: 950-8.

7. Available at: https://ginasthma.org/wp-content/uploads/2018/04/wms-GINA-2018-report-V1.3-002.pdf

8. Ustawa z dnia 23 marca 2017 r. o zmianie ustawy o świadczeniach opieki zdrowotnej finansowanych ze środków publicznych (Dz. U. 2017 poz. 844).

9. Getzen TE. Ekonomika Zdrowia. Koordynowana opieka zdrowotna. Wydawnictwo Naukowe PWN, Warsaw 2013. 